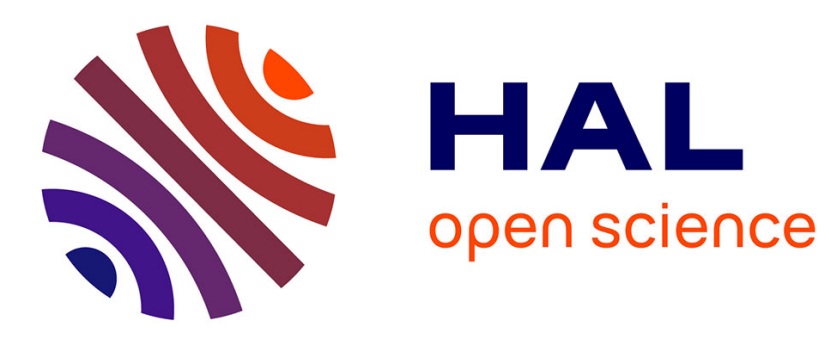

\title{
Asymmetric dispersal in the multi-patch logistic equation
}

Roger Arditi, Claude Lobry, Tewfik Sari

\section{To cite this version:}

Roger Arditi, Claude Lobry, Tewfik Sari. Asymmetric dispersal in the multi-patch logistic equation. Theoretical Population Biology, 2018, 120, pp.11 - 15. 10.1016/j.tpb.2017.12.006 . hal-01688305

\section{HAL Id: hal-01688305 https://hal.sorbonne-universite.fr/hal-01688305}

Submitted on 19 Jan 2018

HAL is a multi-disciplinary open access archive for the deposit and dissemination of scientific research documents, whether they are published or not. The documents may come from teaching and research institutions in France or abroad, or from public or private research centers.
L'archive ouverte pluridisciplinaire HAL, est destinée au dépôt et à la diffusion de documents scientifiques de niveau recherche, publiés ou non, émanant des établissements d'enseignement et de recherche français ou étrangers, des laboratoires publics ou privés. 


\title{
Asymmetric dispersal in the multi-patch logistic equation
}

\author{
Roger Arditi ${ }^{\mathrm{a}, \mathrm{b}, *}$, Claude Lobry ${ }^{\mathrm{c}}$, Tewfik Sari ${ }^{\mathrm{d}, \mathrm{e}}$ \\ a University of Fribourg, Department of Biology, Chemin du \\ Musée 10, 1700 Fribourg, Switzerland. E-mail: roger.arditi@unifr.ch \\ ${ }^{\mathrm{b}}$ Sorbonne Université, Institute of Ecology and Environmental \\ Sciences (iEES-Paris), 75252 Paris cedex 05, France \\ ${ }^{c}$ Université Nice-Sophia-Antipolis, Centre de recherches en histoire \\ des idées (CRHI), 98 boulevard Édouard Herriot, BP 3209, \\ 06204 Nice cedex, France. E-mail: lobrinria@wanadoo.fr \\ dITAP, Irstea, Montpellier SupAgro, Univ Montpellier, \\ Montpellier, France. E-mail: tewfik.sari@irstea.fr \\ e Université de Haute Alsace, LMIA, Mulhouse, France \\ *Corresponding author
}

Key words: Fragmented habitat; migration rate; logistic growth;

carrying capacity

December 19, 2017

\begin{abstract}
The standard model for the dynamics of a fragmented density-dependent population is built from several local logistic models coupled by migrations. First introduced in the 1970s and used in innumerable articles, this standard model applied to a two-patch situation has never been fully analyzed. Here, we complete this analysis and we delineate the conditions under which fragmentation associated with dispersal is either favorable or unfavorable to total population abundance. We pay special attention to the case of asymmetric dispersal, i.e., the situation in which the dispersal rate from patch 1 to patch 2 is not equal to the dispersal rate from patch 2 to patch 1 . We show that this asymmetry can have a crucial quantitative influence on the effect of dispersal.
\end{abstract}

\section{Introduction}

2 We deal here with population dynamics of a fragmented population. This is a problem

3 with potentially very important applied aspects. For example, in conservation ecology,

4 a standard question is whether a single large refuge is better or worse than several 
small ones, with the objective of maximizing the total population abundance of an endangered species (the SLOSS debate; see, e.g., Hanski, 1999). On the contrary, in the context of pest control, the question is whether a single large field is better or worse than several small ones, with the objective of minimizing the occurence of an insect pest or a plant disease. A huge body of theoretical literature exists around these questions. However, even the simplest and most ancient model still contains unresolved aspects with unsupported generalizations.

The theoretical paradigm that has been used to treat these questions is that of a single population fragmented into two coupled patches. It is widely accepted to assume that each subpopulation in each patch follows a local logistic law and that the two patches are coupled by density-independent migrations. Freedman and Waltman (1977) were first to propose the following model:

$$
\left\{\begin{array}{l}
\frac{d N_{1}}{d t}=r_{1} N_{1}\left(1-\frac{N_{1}}{K_{1}}\right)+\beta\left(N_{2}-N_{1}\right), \\
\frac{d N_{2}}{d t}=r_{2} N_{2}\left(1-\frac{N_{2}}{K_{2}}\right)+\beta\left(N_{1}-N_{2}\right),
\end{array}\right.
$$

where $N_{i}$ is the population abundance in patch $i$. The parameters $r_{i}$ and $K_{i}$ are respectively the intrinsic growth rate and the carrying capacity in patch $i$ and $\beta$ is the migration rate, assumed to be identical in both directions. All parameters are assumed to be positive.

After Freedman and Waltman (1977), aspects of this model were later studied by DeAngelis et al. (1979) and Holt (1985), and a graphical presentation was given by Hanski (1999, pp. 43-46) in his reference book on metapopulations. More recently, DeAngelis and Zhang (2014), DeAngelis et al. (2016) have brought new developments. We think we were first to publish the full mathematical study of model (1) in Arditi et al. (2015).

A limitation of model (1) is the assumption of symmetric dispersal: the single parameter $\beta$ quantifies the migration rate from patch 1 to patch 2 and from patch 2 to patch 1 . In the present paper, we will expand our first analysis to the case of asymmetric dispersal between patches and we will delineate the conditions under which dispersal can either be favorable or unfavorable to total population abundance.

We denote by $N_{1}^{*}$ and $N_{2}^{*}$ the population abundances at equilibrium. In isolation $(\beta=0)$, each population equilibrates at its local carrying capacity: $N_{i}^{*}=K_{i}$. Freedman and Waltman (1977) analyzed the model in the case of perfect mixing $(\beta \rightarrow \infty)$ and showed that the total equilibrium population, $N_{T}^{*}=N_{1}^{*}+N_{2}^{*}$, is generally different from the sum of the carrying capacities $K_{1}+K_{2}$. Depending on the parameters, $N_{T}^{*}$ can either be greater or smaller than $K_{1}+K_{2}$. For instance, if $r_{1} / K_{1}<r_{2} / K_{2}$ (with $K_{1}<K_{2}$ ), we will have $N_{T}^{*}>K_{1}+K_{2}$, which means that dispersal is favorable with respect to the total equilibrium population. This spectacular result, somewhat paradoxical, has been widely discussed and has led to speculations about the general virtues of patchiness and dispersal.

Freedman and Waltman (1977) only contrasted the situations of perfect isolation and perfect mixing; they did not study the effect of intermediate values of the dispersal parameter $\beta$. This effect was studied by DeAngelis and Zhang (2014), but only in the special case $r_{1} / K_{1}=r_{2} / K_{2}$. In our earlier paper (Arditi et al. 2015), we calculated 
the full set of parameter conditions for which dispersal is favorable or not to total population abundance.

In another paper, Arditi et al. (2016) returned to the simpler case of perfect mixing (i.e., with the migration rate $\beta \rightarrow \infty$ ) in order to compare the properties of Verhulst's and Lotka's formulations of the logistic model in relation with the paradox outlined above (the non-additivity of carrying capacities). In a criticism of this paper, RamosJiliberto and Moisset de Espanés (2017) proposed the following alternative model:

$$
\left\{\begin{array}{l}
\frac{d N_{1}}{d t}=r_{1} N_{1}\left(1-\frac{N_{1}}{K_{1}}\right)+\beta\left(\frac{N_{2}}{K_{2}}-\frac{N_{1}}{K_{1}}\right), \\
\frac{d N_{2}}{d t}=r_{2} N_{2}\left(1-\frac{N_{2}}{K_{2}}\right)+\beta\left(\frac{N_{1}}{K_{1}}-\frac{N_{2}}{K_{2}}\right) .
\end{array}\right.
$$

In this model, the dispersal rate is $\beta / K_{i}$. It is different in each direction: the probability of an individual to leave its patch is inversely proportional to the local carrying capacity. This is known as the Balanced Dispersal Model proposed by McPeek and Holt (1992). Ramos-Jiliberto and Moisset de Espanés (2017) showed that, in this model, the equality $N_{T}^{*}=K_{1}+K_{2}$ is always true. Thus, the model (2) does not present the "perfect mixing paradox": there is strict additivity of carrying capacities.

In their reply to Ramos-Jiliberto and Moisset de Espanés (2017), Arditi et al. (2017) moved beyond the polemical opposition of models (1) and (2) by embedding both of them into the following more general model with differential dispersal:

$$
\left\{\begin{array}{l}
\frac{d N_{1}}{d t}=r_{1} N_{1}\left(1-\frac{N_{1}}{K_{1}}\right)+\beta\left(\frac{N_{2}}{\gamma_{2}}-\frac{N_{1}}{\gamma_{1}}\right), \\
\frac{d N_{2}}{d t}=r_{2} N_{2}\left(1-\frac{N_{2}}{K_{2}}\right)+\beta\left(\frac{N_{1}}{\gamma_{1}}-\frac{N_{2}}{\gamma_{2}}\right) .
\end{array}\right.
$$

As in model 22, the dispersal rate $\left(\beta / \gamma_{i}\right)$ is generally different in each direction. However, this model encompasses both (1) and (2) because model (1) corresponds to the case $\gamma_{1}=\gamma_{2}=1$ and model (2) corresponds to the case $\gamma_{1}=K_{1}, \gamma_{2}=K_{2}$. Note that model (3) is overparameterized in order that it can be written in a symmetric way. Among the parameters $\beta, \gamma_{1}$, and $\gamma_{2}$, two only are independent. With no loss of generality, the ratio $\gamma_{2} / \gamma_{1}$ can be considered as a single parameter. Thus, model (3)'s total number of independent parameters is six, not seven. In this parameterization, $\beta$ quantifies the migration intensity and $\gamma_{2} / \gamma_{1}$ quantifies the migration asymmetry.

Assuming perfect mixing (i.e., $\beta \rightarrow \infty$ ), Arditi et al. (2017) showed that the paradox exhibited by model (1) is a generic property of the more general model 3 . They showed that its absence in model (2) corresponds to a very special case in parameter space, i.e., balanced dispersal. They also showed that a second special case exists for which carrying capacity additivity is observed.

The purpose of the present paper is to perform the mathematical analysis of the general model (3) in the full parameter space. That is, we will consider all finite positive values of $\beta$ and no longer assume perfect mixing as in Arditi et al. (2017). 


\section{Equilibrium analysis}

79 The equilibria of the dynamic model (3) are the solutions of the algebraic system

$$
\left\{\begin{array}{l}
0=r_{1} N_{1}\left(1-\frac{N_{1}}{K_{1}}\right)+\beta\left(\frac{N_{2}}{\gamma_{2}}-\frac{N_{1}}{\gamma_{1}}\right), \\
0=r_{2} N_{2}\left(1-\frac{N_{2}}{K_{2}}\right)+\beta\left(\frac{N_{2}}{\gamma_{2}}-\frac{N_{1}}{\gamma_{1}}\right) .
\end{array}\right.
$$

$$
B=\left(B_{1}, B_{2}\right)=\left(\frac{\left(\gamma_{1} / \gamma_{2}\right) r_{1}+r_{2}}{\left(\gamma_{1} / \gamma_{2}\right) r_{1} / K_{1}+\left(\gamma_{2} / \gamma_{1}\right) r_{2} / K_{2}}, \frac{r_{1}+\left(\gamma_{2} / \gamma_{1}\right) r_{2}}{\left(\gamma_{1} / \gamma_{2}\right) r_{1} / K_{1}+\left(\gamma_{2} / \gamma_{1}\right) r_{2} / K_{2}}\right) .
$$

Adding the two equations gives

$$
r_{1} N_{1}\left(1-\frac{N_{1}}{K_{1}}\right)+r_{2} N_{2}\left(1-\frac{N_{2}}{K_{2}}\right)=0
$$

which is the equation of an ellipse (shown in red in Fig. 1 and the other figures). This ellipse $\mathscr{E}$ passes through the points $(0,0),\left(K_{1}, 0\right),\left(0, K_{2}\right)$, and $\left(K_{1}, K_{2}\right)$. It does not depend on the migration intensity $\beta$ (or on the migration asymmetry $\gamma_{2} / \gamma_{1}$ ).

Solving the first equation in (4) for $N_{2}$ yields a parabola $\mathscr{P}_{\beta}$ of equation $N_{2}=$ $P_{\beta}\left(N_{1}\right)$, where the function $P_{\beta}$ is defined by

$$
P_{\beta}\left(N_{1}\right)=\gamma_{2}\left(\frac{N_{1}}{\gamma_{1}}-\frac{r_{1}}{\beta} N_{1}\left(1-\frac{N_{1}}{K_{1}}\right)\right) .
$$

This parabola $\mathscr{P}_{\beta}$ (shown in blue in Fig. 1 and the other figures) depends on the migration intensity $\beta$ (and on $\gamma_{2} / \gamma_{1}$ ). It always passes through the points 0 and $\Omega=$ $\left(K_{1}, K_{1} \gamma_{2} / \gamma_{1}\right)$

The equilibria are the nonnegative intersections of the ellipse $\mathscr{E}$ and the parabola $\mathscr{P}_{\beta}$. There are two equilibrium points. The first is the trivial point $(0,0)$ and the second is a nontrivial point whose position depends on $\beta$ :

$$
E_{\beta}=\left(N_{1 \beta}^{*}, N_{2 \beta}^{*}\right) .
$$

A straightforward isocline analysis (see Fig. 2) shows that $(0,0)$ is always unstable and that $E_{\beta}$ is always stable.

When $\beta \rightarrow 0$, the left branch of the parabola $\mathscr{P}_{\beta}$ merges into the vertical line $N_{1}=0$ and the right branch into the vertical line $N_{1}=K_{1}$ ( $\mathscr{P}_{0}$ in Fig. 1). The parabola's limit for $\beta \rightarrow \infty$ is the oblique line $N_{2}=\left(\gamma_{2} / \gamma_{1}\right) N_{1}$ ( $\mathscr{P}_{\infty}$ in Fig. 1 ).

We denote by $A$ the intersection of the ellipse $\mathscr{E}$ with $\mathscr{P}_{0}$ and by $B$ the intersection of $\mathscr{E}$ with $\mathscr{P}_{\infty} . A=\left(K_{1}, K_{2}\right)$ is the perfect-isolation equilibrium and $B$ is the perfectmixing equilibrium. It is easy to calculate that

The slope of $\mathscr{P}_{\infty}$ is $B_{2} / B_{1}$. With the expressions in $(7)$, this slope is found to be $\gamma_{2} / \gamma_{1}$. As this ratio can vary from 0 to $\infty, B$ can be anywhere on the ellipse $\mathscr{E}$ in the positive quadrant. As $\beta$ increases from 0 to $\infty$, the equilibrium point $E_{\beta}$ follows the ellipse arc from $A$ to $B$. This change is clockwise if $\gamma_{2} / \gamma_{1}<K_{2} / K_{1}$ or counterclockwise if $\gamma_{2} / \gamma_{1}>K_{2} / K_{1}$ (respectively left and right panels of Fig. 1 ). 

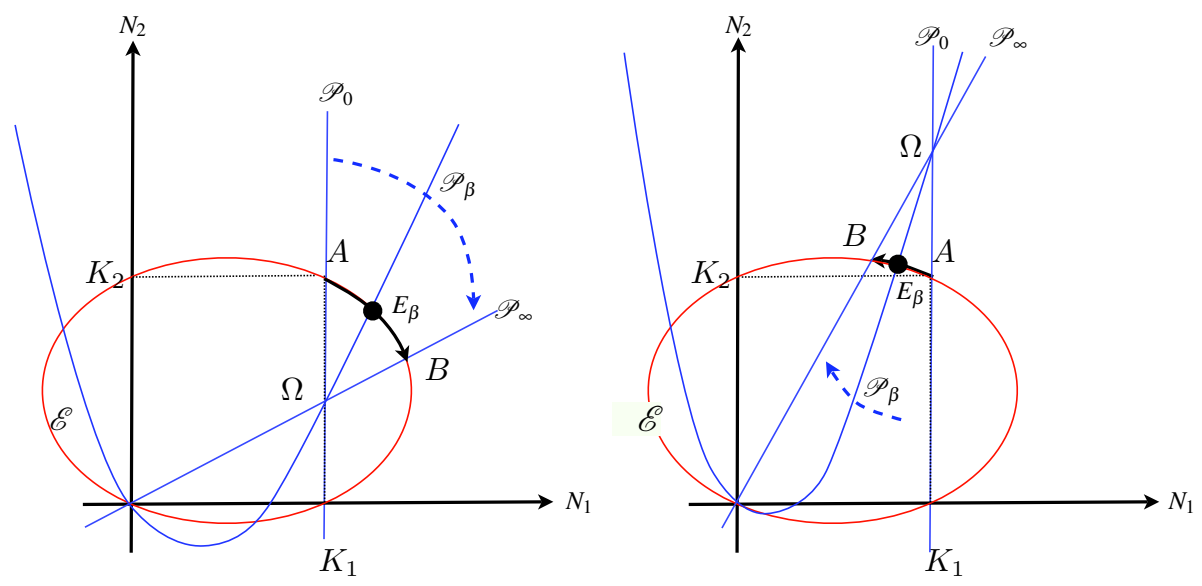

Figure 1: The equilibrium point $E_{\beta}$ is the positive intersection of the ellipse $\mathscr{E}$ and the parabola $\mathscr{P}_{\beta}$. The lines $\mathscr{P}_{0}$ and $\mathscr{P}_{\infty}$ are the limits of $\mathscr{P}_{\beta}$ for $\beta=0$ and for $\beta \rightarrow \infty$. The slope of $\mathscr{P}_{\infty}$ is $\gamma_{2} / \gamma_{1}$. The equilibrium $E_{\beta}$ can only belong to the ellipse arc between $A$ and $B$. Left: $\gamma_{2} / \gamma_{1}<K_{2} / K_{1}$. Right: $\gamma_{2} / \gamma_{1}>K_{2} / K_{1}$.

In the special case $\gamma_{2} / \gamma_{1}=K_{2} / K_{1}$, the points $A$ and $B$ become confounded and the equilibrium $E_{\beta}$ does not depend on $\beta$; it is always equal to $\left(K_{1}, K_{2}\right)$ and thus $N_{T}^{*}=$ $K_{1}+K_{2}$. This is the special case considered by Ramos-Jiliberto and Moisset de Espanés (2017). Note that this occurs in wider conditions than those assumed by these authors: $\beta$ can have any value, not only $\beta \rightarrow \infty$, and it is not necessary to have the separate equalities $\gamma_{1}=K_{1}, \gamma_{2}=K_{2}$; the condition is only on the ratio: $\gamma_{2} / \gamma_{1}=K_{2} / K_{1}$. Anyway, this special case is by no means a representative of the general case.

\section{Influence of dispersal on total population size}

In the previous section, we saw that, depending on the values of the migration parameters $\beta$ and $\gamma_{2} / \gamma_{1}$, the equilibrium can be anywhere on the ellipse $\mathscr{E}$ in the positive quadrant. In this section, we will describe how this position affects the total equilibrium population $N_{T}^{*}$ of model (3). In particular, we will investigate whether $N_{T}^{*}$ is greater or smaller than $K_{1}+K_{2}$. The analysis can largely be done graphically.

On Fig. 3, the straight line $\Delta$ is the line of slope -1 passing through the point $A=\left(K_{1}, K_{2}\right)$. It is the set of points with $N_{1}+N_{2}=K_{1}+K_{2}$. For any equilibrium $E=\left(N_{1}^{*}, N_{2}^{*}\right)$, the total population $N_{T}^{*}=N_{1}^{*}+N_{2}^{*}$ can be read on the intersection with the horizontal axis of the straight line of slope -1 passing through $E$. We see very 

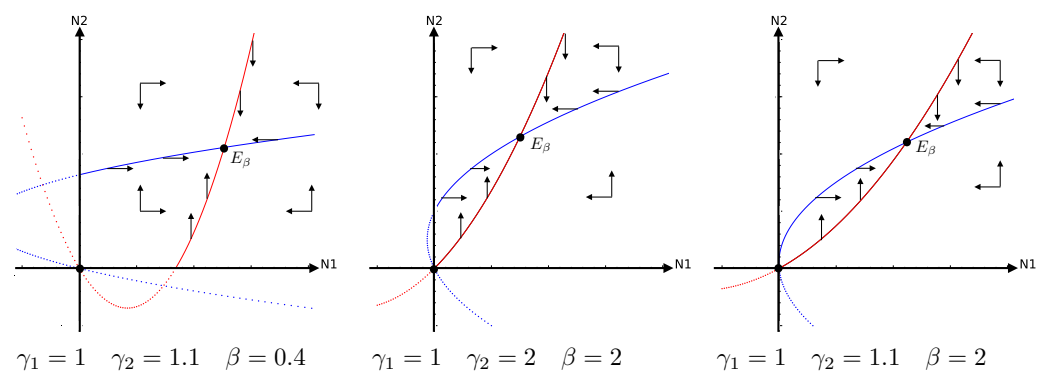

Figure 2: The isoclines of (3) are drawn (in red for $N_{1}$, in blue for $N_{2}$ ) for the parameter values $r_{1}=1, r_{2}=2, K_{1}=1.4, K_{2}=2$ and for three different combinations of migration parameters. These three examples are typical of all possible configurations. In all cases the trajectories are attracted by $E_{\beta}$.

simply that dispersal is favorable to $N_{T}^{*}$ if $E$ is above $\Delta$, unfavorable if below $\Delta$. For example, on Fig. 3. dispersal is favorable when the equilibrium is $E_{\beta_{1}}$ and unfavorable when the equilibrium is $E_{\beta_{2}}$.

Let us consider the slope of the ellipse $\mathscr{E}$ at point $A=\left(K_{1}, K_{2}\right)$. By differentiating the ellipse equation (5) with respect to $N_{1}$, it is easy to calculate that this slope is equal to $-r_{1} / r_{2}$.

In the special case $r_{1}=r_{2}$, the slope is precisely -1 , which means that the ellipse $\mathscr{E}$ is entirely below the straight line $\Delta$ except for the point $A$, which is exactly on $\Delta$. This result can be stated as the following proposition:

Proposition 1 If $r_{1}=r_{2}$, dispersal is always unfavorable to $N_{T}^{*}$.

When $r_{1} \neq r_{2}$, we will assume, with no loss of generality, that $r_{1}<r_{2}$ (as in Fig. 3). In this case, the point $A$ is still an intersection of $\mathscr{E}$ with $\Delta$ but there exists a second intersection point, which we denote by $C$ (see Fig. 33).

We denote by $\Sigma$ the straight line joining the origin to $C$ and by $\sigma$ the slope of $\Sigma$. An easy calculation shows that the coordinates of $C$ are:

$$
C=\left(\frac{r_{2} K_{1}\left(K_{1}+K_{2}\right)}{r_{1} K_{2}+r_{2} K_{1}} \quad, \quad \frac{r_{1} K_{2}\left(K_{1}+K_{2}\right)}{r_{1} K_{2}+r_{2} K_{1}}\right)
$$

meaning that the slope $\sigma$ is:

$$
\sigma=\frac{r_{1}}{r_{2}} \frac{K_{2}}{K_{1}}
$$

The rest of this section is essentially a comment of Figs. 4 and 5 . We saw in Section 2 that the equilibrium $E_{\beta}$ follows the ellipse arc $A B$ when $\beta$ varies from 0 to $\infty$, where $B$ is the intersection of the ellipse $\mathscr{E}$ with the oblique line $\mathscr{P}_{\infty}$. Since the slope of $\mathscr{P}_{\infty}$ 


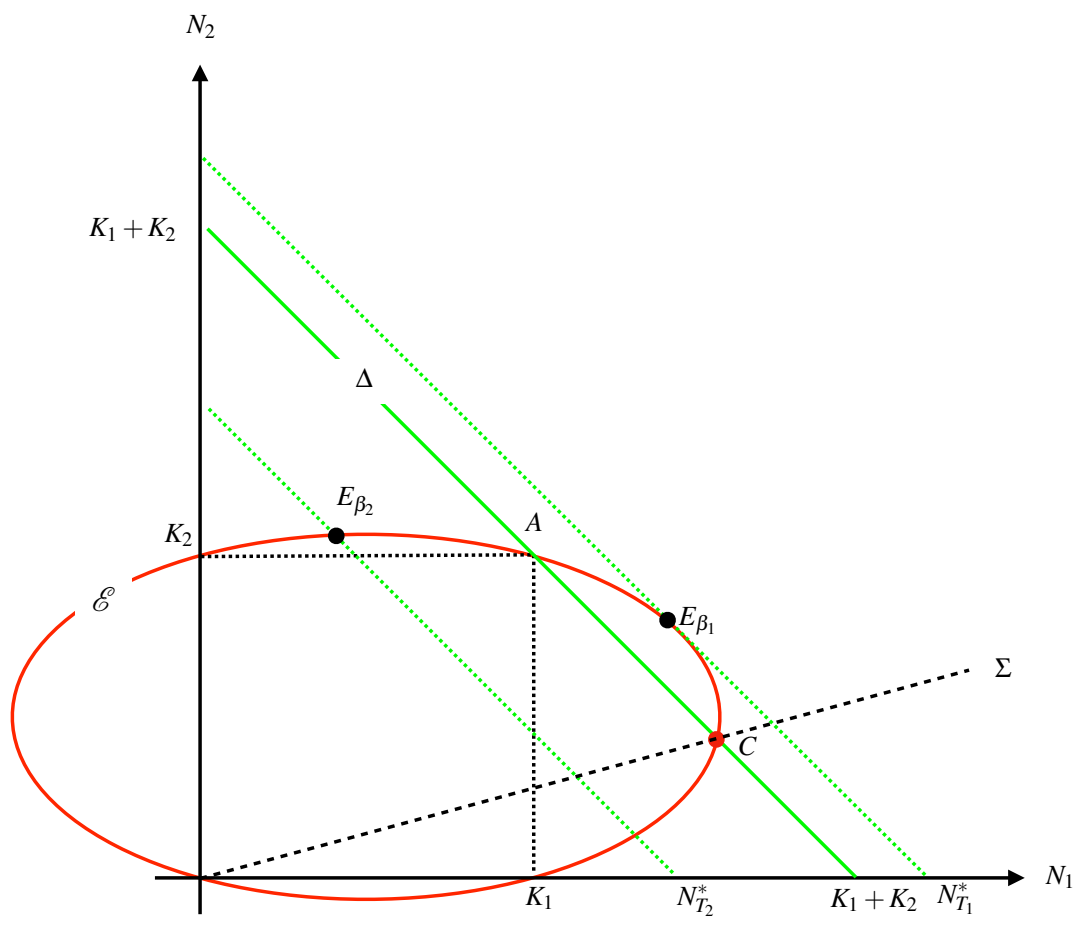

Figure 3: The straight line $\Delta$ is the set of points with $N_{1}+N_{2}=K_{1}+K_{2} . E_{\beta_{1}}$ is an example equilibrium point for which dispersal is favorable, while $E_{\beta_{2}}$ is an example of unfavorable dispersal.

is $\gamma_{2} / \gamma_{1}$, we will distinguish the following three cases, as this slope increases:
(a) $\frac{\gamma_{2}}{\gamma_{1}}<\frac{r_{1}}{r_{2}} \frac{K_{2}}{K_{1}}$,
(b) $\frac{r_{1}}{r_{2}} \frac{K_{2}}{K_{1}} \leq \frac{\gamma_{2}}{\gamma_{1}}<\frac{K_{2}}{K_{1}}$,
(c) $\frac{K_{2}}{K_{1}} \leq \frac{\gamma_{2}}{\gamma_{1}}$.

Figure 4 presents the case (a), in which $\mathscr{P}_{\infty}$ is lower than $\Sigma$. Figure 5 presents the other two cases, with $\mathscr{P}_{\infty}$ higher than $\Sigma$ but lower than $A$ (case b) and $\mathscr{P}_{\infty}$ higher than $A$ (case c). Besides each of the pictures in the state space $N_{1} \times N_{2}$, we show a qualitative graph of the function $\beta \mapsto N_{T}^{*}(\beta)$.

Let us first consider the case (a) on Fig. 4 For $\beta=0$, the equilibrium point starts at $A$ and, as $\beta$ increases, $E_{\beta}$ moves clockwise along $\mathscr{E}$ and ends at $B$. The total equilibrium population $N_{T}^{*}(\beta)$ starts with the value $K_{1}+K_{2}$ at $A$, then increases, attains a maximum $N_{\text {max }}^{*}$ for some $\beta_{\max }$, decreases to $K_{1}+K_{2}$ again at point $C$ for some $\beta_{C}$ and decreases further to the limit corresponding to point $B$. Note that $N_{\max }^{*}, \beta_{\max }, \beta_{C}$, and $B$ can all be calculated explicitly but we will not give them here because the expressions are heavy and have no practical interest. 

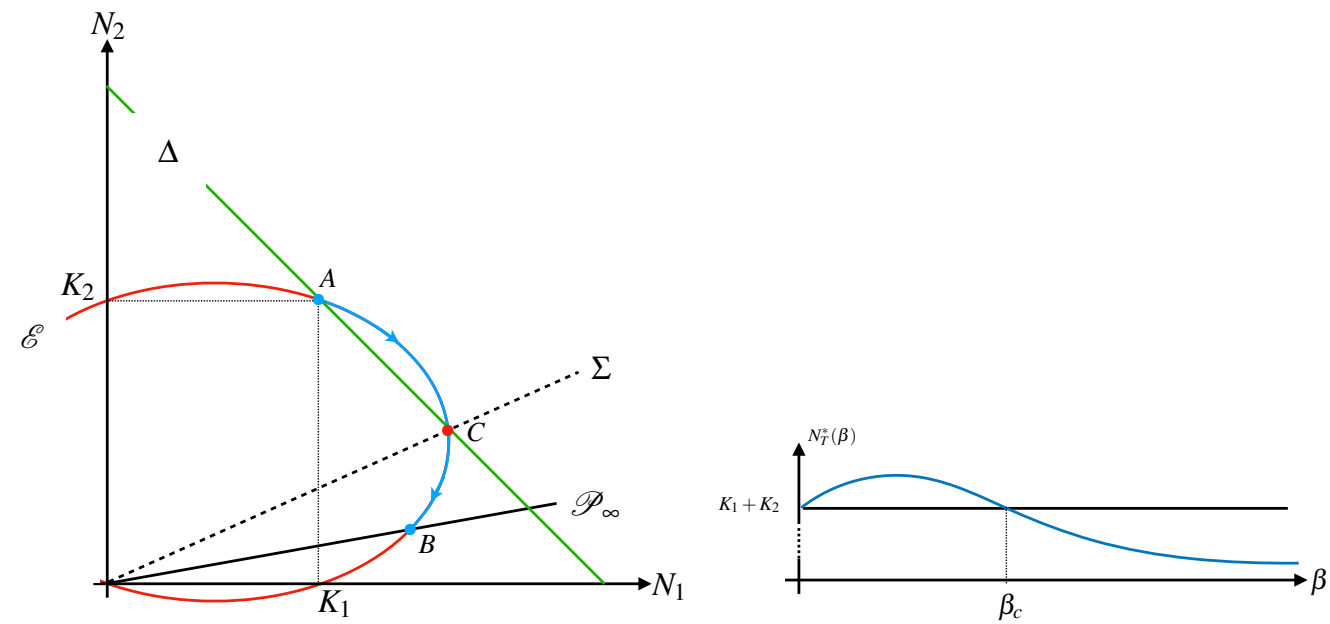

Figure 4: This illustrates the case (a) of 10 . As the migration intensity $\beta$ increases from 0 to $\infty$, the equilibrium point moves clockwise along the ellipse $\mathscr{E}$ from $A$ to $B$, passing through $C$.

For the other two cases (Fig. 5), descriptions are similar but simpler because $N_{T}^{*}(\beta)$ is either always greater than $K_{1}+K_{2}$ (case b) or always smaller than $K_{1}+K_{2}$ (case c).

This description can be summarized in the following proposition.

Proposition 2 Assume that $r_{1}<r_{2}$. Then:

(a) $\frac{\gamma_{2}}{\gamma_{1}}<\frac{r_{1}}{r_{2}} \frac{K_{2}}{K_{1}} \Longrightarrow$ there exists $\beta_{C}$ such that

$$
\begin{aligned}
& 0 \leq \beta \leq \beta_{C} \Longrightarrow N_{T}^{*}(\beta) \geq K_{1}+K_{2}, \\
& \beta_{C}<\beta \Longrightarrow N_{T}^{*}(\beta)<K_{1}+K_{2},
\end{aligned}
$$

(b) $\frac{r_{1}}{r_{2}} \frac{K_{2}}{K_{1}} \leq \frac{\gamma_{2}}{\gamma_{1}}<\frac{K_{2}}{K_{1}} \Longrightarrow N_{T}^{*}(\beta) \geq K_{1}+K_{2}$ for every $\beta$,

(c) $\frac{K_{2}}{K_{1}} \leq \frac{\gamma_{2}}{\gamma_{1}} \Longrightarrow N_{T}^{*}(\beta) \leq K_{1}+K_{2}$ for every $\beta$.

\section{Discussion}

The ecological problem that has motivated this study is to find the conditions for which fragmentation and dispersal can lead to higher total equilibrium population abundance $N_{T}^{*}$ than the sum $K_{1}+K_{2}$. Mathematically, this is the six-parameter problem posed by model (3) that we have solved in the present paper.

The propositions 1 and 2 contain the full set of results of the present general model (3). They show that all parameters have an influence in determining whether $N_{T}^{*}$ is 

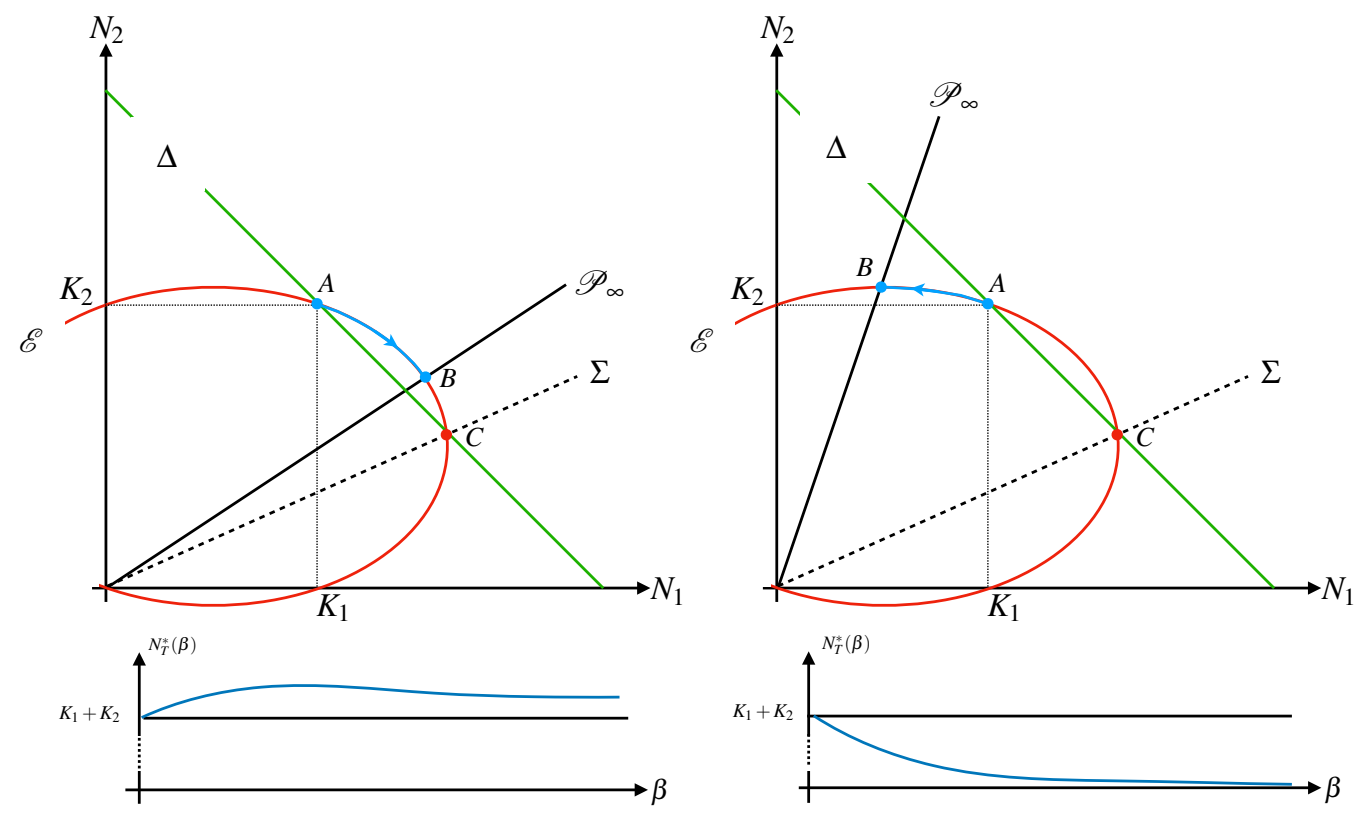

Figure 5: Left: case (b) of (10). As the equilibrium point moves clockwise from $A$ to $B$ with increasing $\beta$, it is always greater than $K_{1}+K_{2}$. Right: case (c) of 10$]$. As the equilibrium point moves counterclockwise from $A$ to $B$ with increasing $\beta$, it is always smaller than $K_{1}+K_{2}$.

higher or lower than $K_{1}+K_{2}$. Compared with earlier models, which new results are brought by dispersal asymmetry? This can be found by investigating the influence of $\gamma_{2} / \gamma_{1}$ in the two propositions, and by considering the special value $\gamma_{2} / \gamma_{1}=1$ that corresponds to symmetric dispersal.

Proposition 1 does not depend on $\gamma_{2} / \gamma_{1}$ and remains valid in the case of symmetric dispersal: dispersal is always unfavorable when $r_{1}=r_{2}$.

In Proposition 2 for $r_{1}<r_{2}$, the assumption of symmetric dispersal simplifies the conditions for the three cases, which become:

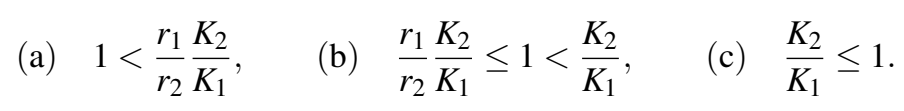

As an example, let us have a closer look at condition (c). If this condition is satisfied, dispersal always has an unfavorable effect on total abundance, for any dispersal intensity $\beta$. If dispersal is symmetric, the inequality (11-) means that (with $r_{1}<r_{2}$ ), the total equilibrium abundance $N_{T}^{*}$ will always be lower than $K_{1}+K_{2}$ when $K_{2} \leq K_{1}$. However, in the presence of dispersal asymmetry, the corresponding condition (10) is not necessarily satisfied: if the asymmetry is such that $\gamma_{2} / \gamma_{1} \ll 1$, dispersal can become favorable. Conversely, if $\gamma_{2} / \gamma_{1} \gg 1$, dispersal remains unfavorable in wider conditions 
of the ratio $K_{2} / K_{1}$. Similarly, the conditions $(10$ a) and $(10 \mathrm{p})$ are also influenced by the asymmetry $\gamma_{2} / \gamma_{1}$.

In sum, dispersal asymmetry can play a crucial role. The various patterns describing the influence of dispersal on total population abundance (the small graphs in Figs.4 and 5) remain qualitatively the same whether dispersal is symmetric or not. However, comparing the conditions (10) and (11) shows that dispersal asymmetry can have a strong quantitative influence, depending on its magnitude and on its direction. In combination with the other parameters, it can either amplify or attenuate the favorable or unfavorable effects of dispersal intensity. Strong asymmetry combined with high dispersal intensity can reverse the predictions of symmetric dispersal. This is particularly important if the model is used for applied purposes such as population conservation or pest control.

\section{References}

[1] Arditi, R., Bersier, L.F., Rohr, R.P., 2016. The perfect mixing paradox and the logistic equation: Verhulst vs. Lotka. Ecosphere 7(11):e01599.

[2] Arditi, R., Bersier, L.F., Rohr, R.P., 2017. The perfect mixing paradox and the logistic equation: Verhulst vs. Lotka: Reply. Ecosphere 8(7):e01894.

[3] Arditi, R., Lobry, C., Sari, T., 2015. Is dispersal always beneficial to carrying capacity? New insights from the multi-patch logistic equation. Theoretical Population Biology 106, 45-59.

[4] DeAngelis, D.L., Ni, W.M., Zhang, B., 2016. Effects of dispersal in a nonuniform environment on population dynamics and competition: a patch model approach. Theoretical Ecology 9, 443-453.

[5] DeAngelis, D.L., Travis, C.C., Post, W.M., 1979. Persistence and stability of seed-dispersed species in a patchy environment. Theoretical Population Biology 16, 107-125. doi:10.1016/0040-5809(79)90008-X.

[6] DeAngelis, D.L., Zhang, B., 2014. Effects of diffusion on total biomass in heterogeneous continuous and discrete-patch systems. Discrete and Continuous Dynamical Systems Series B 19, 3087-3104. doi:10.3934/dcdsb.2014.19.3087.

[7] Freedman, H.I., Waltman, D., 1977. Mathematical models of population interactions with dispersal. I. Stability of two habitats with and without a predator. SIAM Journal of Applied Mathematics 32, 631-648. doi:10.1137/0132052.

[8] Hanski, I., 1999. Metapopulation Ecology. Oxford University Press.

[9] Holt, R.D., 1985. Population dynamics in two-patch environments: Some anomalous consequences of an optimal habitat distribution. Theoretical Population Biology 28, 181-208. doi:10.1016/0040-5809(85)90027-9.

[10] Ramos-Jiliberto, R., Moisset de Espanés, P., 2017. The perfect mixing paradox and the logistic equation: Verhulst vs. Lotka: Comment. Ecosphere 8:e1895. 